\title{
La experiencia de orientación docente en la práctica profesional de trabajo social
}

\author{
Johana Linares García ${ }^{1}$ \\ Andrea Hernández Quirama ${ }^{2}$ \\ Hector Mauricio Rojas Betancur ${ }^{3}$
}

Recibido: 13-01-2020

Aceptado: 12-05-2020

\section{Resumen}

Este artículo es resultado de la sistematización de la experiencia con orientación docente en el marco de la práctica profesional de trabajadores sociales. El proceso de sistematización contiene tres fases: reconstrucción de la experiencia, interpretación y potenciación de la práctica pedagógica, realizada desde el enfoque crítico-social a partir de los resultados del proceso desarrollado con tres experiencias durante un año. De los resultados se destaca el interés de los estudiantes por materializar el proyecto ético y político ideado para la profesión, y las tensiones presentes entre las fuerzas que integran el campo (agentes e instituciones).

\footnotetext{
1. Trabajadora social, Mg. en Ordenamiento Territorial, doctoranda en Servicio Social de la Universidad Federal de Río de Janeiro. Profesora de la Universidad Industrial de Santander, Bucaramanga, Colombia.

Correo electrónico: johana.linares.garcia@gmail.com

ORCID: http://orcid.org/0000-0001-5401-4633

CvLac: http:/scienti.colciencias.gov.co:8081/cvlac/visualizador/generarCurriculoCv. do?cod_rh=0001198378

2. Trabajadora social, Ph. D. en Investigaciones Feministas y Género. Profesora de la Universidad Industrial de Santander, Bucaramanga, Colombia.

Correo electrónico: ahernanq@uis.edu.co

ORCID: http://orcid.org/0000-0003-1955-0566

CvLac: http://scienti.colciencias.gov.co:8081/cvlac/visualizador/generarCurriculoCv. do?cod_rh $=0001211560$

3. Sociólogo, Ph. D. en Ciencias Sociales. Profesor de la Universidad Industrial de Santander, Bucaramanga, Colombia.

Correo electrónico: hmrojasb@uis.edu.co

ORCID: http://orcid.org/0000-0003-0569-8236

CvLac: https://scienti.minciencias.gov.co/cvlac/visualizador/generarCurriculoCv. do?cod_rh $=0000142123$
} 
Además, los estudiantes tienden a regresar al positivismo y con el transcurrir de la práctica se adaptan a la lógica del proyecto educativo mercantilizado, lugar en el que el docente ha contribuido a adoctrinar para el mercado laboral. El mayor reto de la orientación docente se centra en promover al interior de la unidad académica la discusión en torno a las competencias y al sistema de evaluación creado para las prácticas académicas, así como en mantener el análisis constante del contexto sociopolítico contemporáneo a fin de promover el desarrollo de capacidades críticas y reflexivas.

Palabras clave: orientación docente, proyecto ético-político, práctica profesional, intervención social.

\section{The experience of teacher orientation in the professional practice of social work}

\section{Abstract}

This article is the result of the systematization of the experience with teacher guidance within the framework of the professional practice of social workers. The systematization process contains three phases: reconstruction of the experience, interpretation and empowerment of pedagogical practice, carried out from the criticalsocial approach based on the results of the process developed with three experiences during one year. The results highlight the interest of the students in materializing the ethical and political project devised for the profession, and the tensions present between the forces that make up the field (agents and institutions). In addition, students tend to return to positivism and with the passage of practice they adapt to the logic of the commercialized educational project, a place in which the teacher has contributed to indoctrinate for the labor market. The greatest challenge of teaching guidance is focused on promoting within the academic unit the discussion around competences and the evaluation system 
created for academic practices, as well as maintaining constant analysis of the contemporary socio-political context in order to promote the development of critical and reflective capacities.

Keywords: teacher orientation, ethical-political project, professional practice, social intervention.

\section{Introducción}

La profesión de trabajo social se ha caracterizado por vincularse al paradigma sociocrítico, en donde se otorga mayor relevancia a la práctica, a partir de la cual se analizan los medios de producción por cuanto son el reflejo de las relaciones desiguales de poder en la sociedad. En este marco, la formación en investigación en trabajo social adquiere mayor relevancia en la profesión a partir del proceso de reconceptualización. Durante este periodo (años 70's), la reflexividad sobre la intervención se convirtió en uno de los ejes de la discusión profesional que se centró en su fundamentación teórica y metodológica. Descolonizar el conocimiento sería posible por medio de la incursión de los trabajadores sociales en el ámbito de la investigación académica, pues la acción profesional razonada otorgaría la voz a los actores, permitiría pensar el mundo desde los de abajo, los oprimidos, los marginados, para cuestionar así las estructuras de poder dominantes y trascender de ser simples ejecutores de políticas sociales a agentes de cambio. En palabras de Netto,

la principal conquista del Movimiento de Reconceptualización fue el rechazo de los asistentes sociales a caracterizarse exclusivamente como agentes técnicos ejecutores de las políticas sociales. A través del proceso de recalificación, principalmente con el ingreso de estos profesionales en el ámbito de la investigación académica, fue posible romper con la división establecida de trabajo entre científicos sociales (los teóricos) y asistentes sociales 
(los profesionales de la práctica). (Netto, 2005, citado por Jara, 2018, p. 5)

Esto implicó, entre otras cuestiones, repensar el currículo de formación profesional y enseñar a hacer ciencia; y conllevó asimismo definir el proyecto ético-político profesional para hacer frente al contexto neoliberal (Montaño, 2007). En este punto, la orientación docente universitaria a partir de prácticas pedagógicas integradoras se convierte en uno de los elementos fundamentales para alcanzar estos propósitos, al permitir potenciar las capacidades de los profesionales en formación, a fin de que logren vincularse a las culturas discursivas de su profesión, cuestionarlas, fundamentarlas y transformarlas.

Por tal razón, en los currículos se da fuerza a la formación en investigación y a la subordinación de los métodos en la práctica para leer las realidades sociales en las que se insertan los profesionales. No obstante, la profesión se ha encapsulado a sí misma al considerar como proceder científico exclusivamente aquel basado en el método positivista (Parola, 2009); allí el docente y el currículo permiten mantener la segmentación de la realidad en campos, fomentando así la dualidad entre la teoría y la práctica. Se enseña que la realidad es susceptible de ser acomodada bajo los cánones de la ciencia, que, siendo rigurosa, relega la reflexividad con, desde y para la gente.

La presente disertación presenta los retos de la docencia durante las prácticas preprofesionales a partir de la sistematización de la experiencia personal como orientadora en una de las escuelas de trabajo social más representativas del nororiente colombiano. Para el caso, el escenario de práctica es el último espacio académico del área disciplinar en donde los estudiantes tienen la oportunidad de confrontar los enfoques epistemológicos, teórico-conceptuales y metodológicos para posibilitar procesos de intervención. No obstante, a pesar de los intereses por desarrollar procesos prácticoparticipativos, el currículo obliga a positivizar la intervención y el docente se encarga entonces de perpetuar las construcciones hegemónicas del conocimiento. 
Este ejercicio de reflexión plantea, a partir de la reconstrucción de la experiencia, pensar la práctica docente desde el campo de fuerzas que se da durante la orientación entre los agentes (estudiantes) y la institucionalidad (escenarios de intervención y la universidad). Para ello se hace necesario cuestionar el habitus, dado que este, como producto de la historia, es un sistema abierto a la experiencia que puede ser perdurable pero no inmutable (Bourdieu y Wacquant, 2005, p. 195).

A continuación, se presentan entonces los aspectos metodológicos de la sistematización de la experiencia que estuvo compuesta por tres fases desarrolladas posteriormente: la reconstrucción, la interpretación desde la docente y las estudiantes, y la potenciación, momento en que se plantean los retos para reorientar el proceso de enseñanza-aprendizaje en las prácticas preprofesionales.

\section{Aspectos metodológicos}

La reflexión se realiza desde el paradigma crítico-social, y es elaborada a partir de la sistematización de la experiencia docente durante el año en curso, en el marco de la orientación de las prácticas profesionales de tres estudiantes vinculadas a una organización no gubernamental que adelanta procesos de defensa del territorio en el Magdalena Medio colombiano.

Sistematizar la experiencia implica realizar no solo la descripción de las fuerzas que se encuentran en la intervención del docente orientador, sino que, a su vez, comprende un esfuerzo analítico que implica

asumir la sistematización de experiencias como el referente desde donde podemos reflexionar sobre los diversos aspectos que se encuentran en una experiencia: resignificaciones, recontextualización, transformaciones - discursivas y prácticas-, trama de relaciones - tensiones, conflictos - y escenario - campo de fuerzas-, con 
el ánimo de trascender la mirada victimizadora y visibilizar lo potencial de nuestro desempeño. (Bermúdez, 2011, p. 99)

Por lo anterior, el proceso está compuesto por tres fases: reconstrucción, interpretación y potenciación de la experiencia (Acevedo, 2008). La primera fase implica la descripción del proceso de orientación, la segunda lleva a comprender los significados atribuidos a la experiencia por parte de la docente y las estudiantes y, por último, la visibilización de los elementos que permiten la transformación de las prácticas educativas.

Las técnicas para la gestión de la información son la observación participante, los grupos de discusión grupal en torno a la intervención de las estudiantes y la orientación docente, así como las sesiones de acompañamiento individual. Los instrumentos utilizados para el registro de la información son los diarios de campo de las estudiantes y la docente, y las memorias de las sesiones de encuentro grupal e individual.

En total, se analizaron treinta y cinco diarios de campo, ocho memorias colectivas y quince memorias de la orientación individual. Esta información fue codificada indicando el tipo de instrumento, el número consecutivo, la fecha y los datos de la persona que lo produce, tal y como se muestra en la figura 1.

Figura 1. Codificación de los insumos.

\section{DC1-08042019-Estudiante 1}

Tipo de instrumento. Para el caso se usó: DC : Diario de campo MC : Memorias de sesiones colectivas MI: Memorias orientación individual

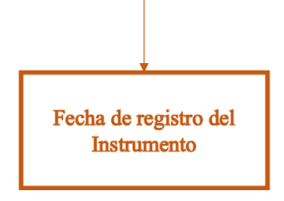

Código asignado a cada estudiante y a la docente. Para el caso puede ser: Estudiante 1 / Estudiante 2 / Estudiante 3 Docente Orientadora

Fuente: elaboración propia. 
Una vez organizada la información, se procede a su análisis e interpretación (Rodríguez, Lorenzo y Herrera, 2005). Para tal fin, se realiza la reducción de los datos, que consiste en un ejercicio de categorización buscando revelaciones en los textos sobre la experiencia de la docente y las estudiantes, teniendo en cuenta los contextos, las tensiones, las relaciones y los significados. Luego de ello se crean relaciones entre las categorías inductivas y la deductiva, y finalmente, para la obtención de resultados y conclusiones, se contrastan los hallazgos con elementos teórico-conceptuales sobre la intervención profesional, especialmente de origen latinoamericano. Es importante mencionar en este punto que los resultados de este artículo se obtienen luego de realizar una reflexión conjunta con las estudiantes participantes.

\section{Reconstrucción de la experiencia}

Para reconstruir la experiencia se presenta a continuación el contexto institucional de la práctica académica, seguido de la descripción de la secuencia didáctica, en donde se da cuenta de la metodología seguida por la docente durante el año en curso; y finalmente se presenta la experiencia como un ejercicio de descripción sobre las acciones de acompañamiento al estudiante.

\section{La práctica académica: el currículo}

La práctica profesional está pensada en la Escuela de Trabajo Social como un espacio de formación con una duración de dos semestres académicos en los niveles 9 y 10, en los cuales los estudiantes deben adelantar las fases del proceso operativo de intervención profesional. De esta manera, la práctica hace parte del área disciplinar de formación profesional y se ubica en el último año académico, cuenta con una intensidad horaria de 20 horas, y el requisito para matricularla es haber aprobado el curso de Sistematización de Experiencias que se imparte en el octavo nivel. 
Durante la Práctica I, el estudiante, con el acompañamiento del docente, debe plantearse tres cuestionamientos básicos:

1) ¿Qué enfoques epistemológicos, teórico-conceptuales y metodológicos posibilitan procesos de intervención profesional de trabajo social para la promoción, ejercicio y garantía de los derechos humanos y el abordaje del conflicto social?, 2) ¿cómo analizar e interpretar la realidad social desde el enfoque de derechos, la perspectiva de género, el enfoque diferencial, étnico y de desarrollo sostenible? y 3) ¿cuál es el proyecto ético político que sustenta la intervención profesional del Trabajador social en el marco de los Derechos Humanos? (Escuela de Trabajo Social, 2011, p. 419)

Para tal fin, se propone la construcción de cuatro informes que deben dar cuenta del proceso: el primero se basa en la ubicación institucional, momento en que los estudiantes dan cuenta del conocimiento de la estructura organizativa de la institución o entidad en donde realizarán su intervención, los cuales son conocidos como los ámbitos, e identifica su posible objeto de intervención; el segundo constituye un esfuerzo de fundamentación teórica de este objeto que debe ser retroalimentado y ajustado a lo largo de toda la práctica profesional; el tercero es un ejercicio diagnóstico en el cual se acude a la investigación como herramienta metodológica para la identificación de una situación problema y la concreción de su objeto de intervención; y el cuarto es un esfuerzo de planificación que debe ser coherente tanto con el referente teórico-conceptual como con el tipo de diagnóstico utilizado.

Culminado este ciclo, se está listo para la ejecución de la propuesta, la cual se desarrolla en el marco de la Práctica II (nivel 10), en donde aborda otros cuestionamientos:

¿Cómo desarrollar la capacidad reflexiva sobre la realidad social?, ¿cuáles son las estrategias de fortalecimiento del tejido social?, ¿cuál es la situación de derechos humanos de los y las ciudadanas y cómo promover su ejercicio?, 
¿qué enfoques teóricos, conceptuales y metodológicos se requieren para el análisis e identificación de alternativas para el abordaje del conflicto social?, ¿a partir de qué axiomas se construye la ética profesional?, ¿cómo se operacionaliza la responsabilidad social empresarial y cuál es su sentido ético?, ¿cómo configurar nuevas subjetividades para el cambio social?, ¿qué retos y tendencias demandan los problemas sociales contemporáneos en la intervención de trabajo social? (Escuela de Trabajo Social, 2011, p. 425)

Asimismo, al igual que en la Práctica I, en este espacio el estudiante debe dar cuenta del proceso mediante tres informes: el primero será para afinar el referente teórico-conceptual acorde a su problema u objeto de intervención; el segundo realiza un balance de la ejecución, es decir, describe las actividades realizadas para los objetivos planteados; y finalmente, el tercer informe consiste en la evaluación, momento en el que presenta los logros, dificultades y retos de su formación profesional.

Pero en estos escenarios, ¿cuál es el rol del docente? Actualmente, este asunto se halla en discusión al interior de la Escuela de Trabajo Social de la institución; para algunos profesores su rol es el de supervisar al estudiante y para otros es el de orientar en la toma de decisiones. Si bien es cierto que existe la libertad de cátedra, se deben seguir los parámetros establecidos en el proyecto educativo del programa y cumplir con las evaluaciones programadas de la práctica; para ello se reconocen salarialmente 20 horas por estudiante/mes, que se deben distribuir entre seminarios y reuniones, visitas a las instituciones o ámbitos, revisión de informes y encuentros pedagógicos y de asesoría individual.

\section{La secuencia didáctica: metodología planeada para orientar la práctica profesional en trabajo social}

El aprendizaje, como proceso, plantea al docente la necesidad de construir secuencias didácticas para lograr desarrollar o fortalecer 
determinados comportamientos (hoy denominados competencias) que permitan al estudiante, más allá del logro de resultados, reflexionar sobre su actuar y articular el saber con la experiencia. Sin lugar a dudas, la formulación de los principios para orientar la práctica por parte del docente responde en gran medida a la articulación entre las acciones que pretende desarrollar para dar cumplimiento al plan curricular (Díaz, 2013), el cual responde en gran medida a las políticas nacionales de educación, que, para el caso, en Colombia se orientan hacia un aprendizaje conductual que atienda las demandas del mercado. De esta manera, se han precarizado no solo las condiciones de estudio, sino también la calidad en la formación, dado que se reduce la formación a las necesidades del mercado. Actualmente,

No âmbito da política educacional, sobretudo, no que diz respeito à expansão do ensino superior, é inegável que vivemos em um período onde o desmonte da Política de Educação de nível superior vem ocorrendo a passos largos marcados pelos processos de mercantilização, de precarização das condições de trabalho dos docentes e de precarização das condições de estudo; processo este que aponta para uma certificação em larga escala, em que a qualidade da formação vem sendo colocada em questão. (Ramos y Santos, 2016, p. 284)

Esta puede ser considerada la primera tensión entre el proyecto de formación ético político y las demandas institucionales de formación profesional. El docente parte desde el propósito del espacio académico diseñado por la Escuela de Trabajo Social, que en gran medida responde a las necesidades del mercado, para la formulación de competencias cognitivas, procedimentales, axiológicas y actitudinales. A partir de ellas, establece los contenidos, las estrategias de enseñanza y aprendizaje, y el sistema de evaluación.

Durante la práctica profesional se espera que más allá de la reproducción de contenidos, el docente enseñe a aprender a los estudiantes, pues "solamente cuando los estudiantes conozcan 
sus procesos cognitivos y aprendan a regularlos, serán realmente aprendices autónomos" (Corredor, Arbeláez y Pérez, 2009, p. 60).

De igual forma, durante este espacio académico el estudiante conoce el género textual propio del informe de práctica a fin de favorecer su inclusión en el discurso académico propio de su formación. Indudablemente,

familiarizar a los alumnos con una gran variedad de géneros puede resultar de gran utilidad para construir una competencia discursiva que les permita, por un lado, in-

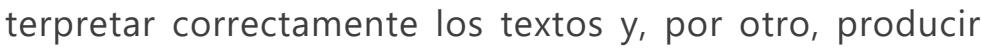
textos de acuerdo con las convenciones socioculturales, estructurales y lingüísticas del género discursivo al que pertenecen. (Alexopoulou, 2011, p. 105)

No obstante, la rigurosidad en los informes genera en ocasiones producción escrita descriptiva y estéril, dado que se siguen estructuras textuales que, aunque cumplen con los requisitos institucionales, dejan de lado el carácter reflexivo del estudiante. Para superar este problema, la orientación docente debe permitir potenciar la escritura como medio para materializar el pensamiento y la lectura, a partir de la selección de bibliografía adecuada al contexto en que se quiere intervenir.

De esta manera, como paso previo al primer encuentro con las estudiantes y acorde a los requisitos institucionales, se selecciona el material básico de lectura que sea pertinente a los campos de intervención; para el caso, las estudiantes se vinculan a procesos de desarrollo comunitario en el campo de ambiente, hábitat humano y territorio, un escenario emergente en la Escuela. Asimismo, se construye un cronograma de trabajo que se ajusta a los tiempos académicos, y se establecen, por acuerdo, las equivalencias cuantitativas para el momento de la intervención; esto se realiza al inicio de las Prácticas I y II. En la tabla 1 se observa un ejemplo de la planeación. 
Tabla 1. Cronograma de actividades de la Práctica II y porcentajes que representan.

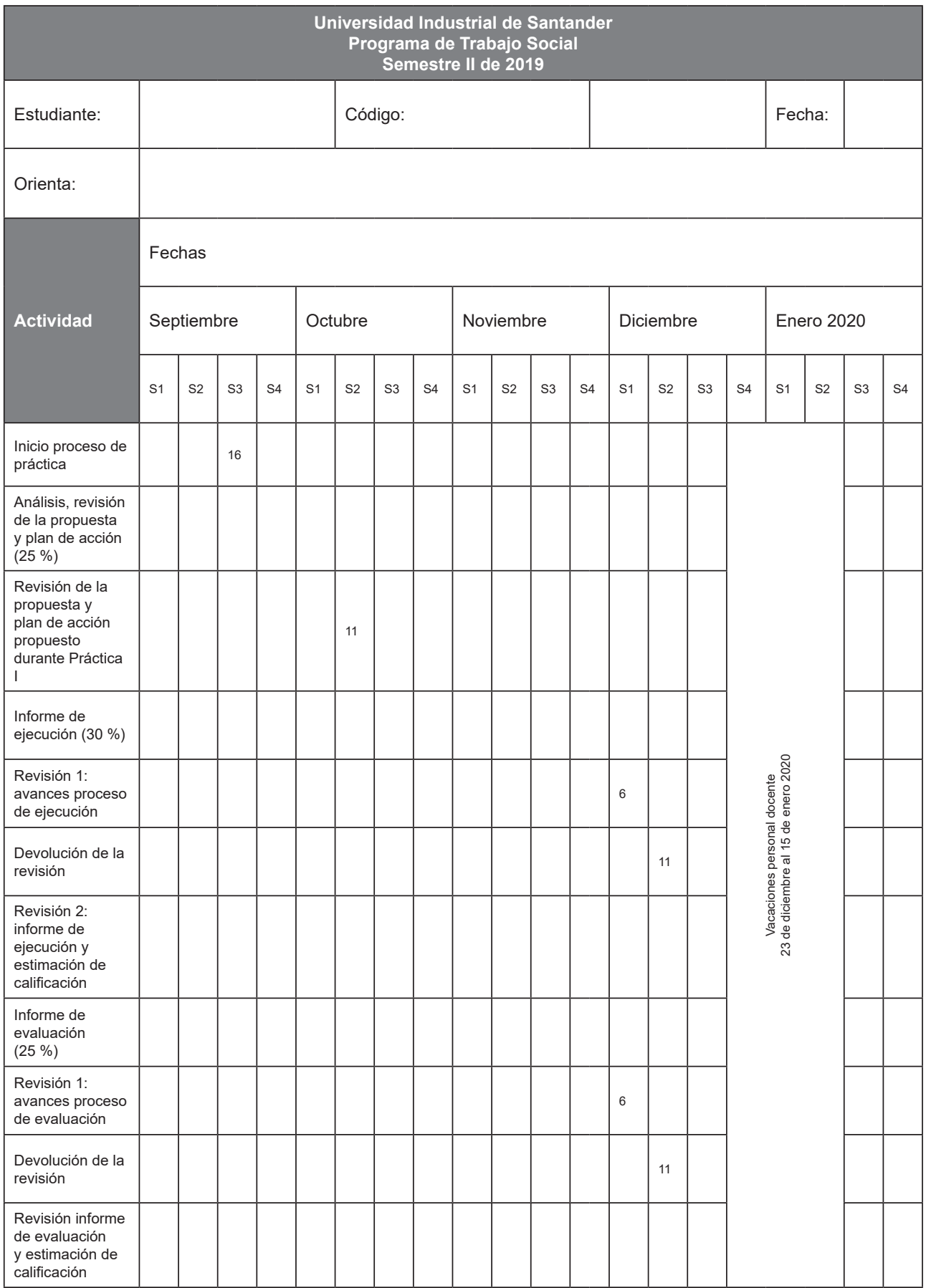




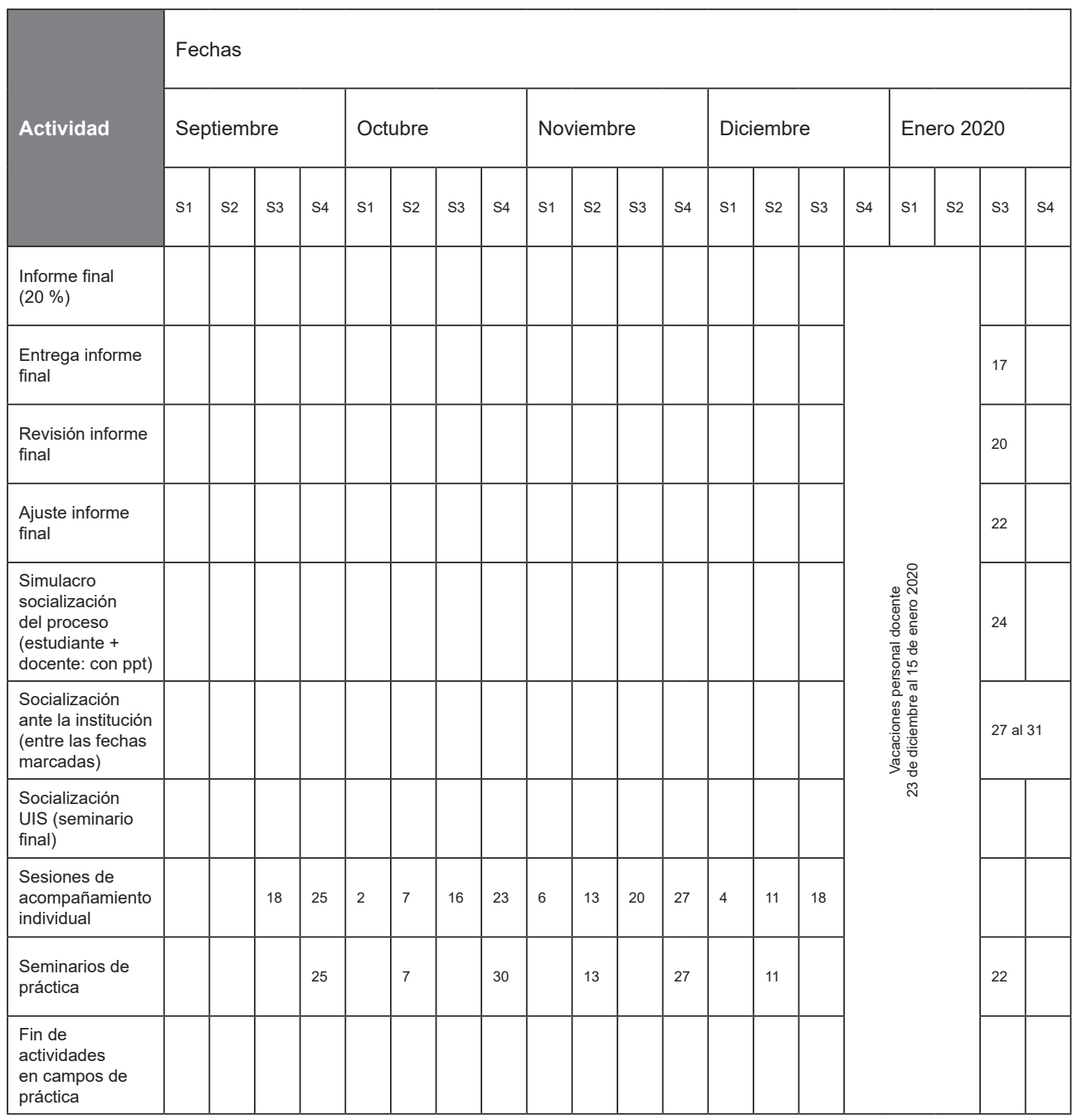

Fuente: elaboración propia.

En el primer encuentro con las estudiantes se escuchan las expectativas del profesional en formación, luego se presentan los propósitos de la práctica, las competencias esperadas, las estrategias docentes para lograrlas, y se establecen los compromisos. Como estrategias didácticas se proponen:

- La creación de un espacio virtual (repositorio) que contenga documentación sobre el proceso de 
intervención (diagnóstico, planeación, ejecución y evaluación), referencias teórico-conceptuales, informes por ámbitos, reflexiones del campo y formatos.

- Encuentros grupales quincenales de reflexión y construcción teórico-metodológica.

- Encuentros semanales de orientación individual.

- Encuentros quincenales grupales de reflexión sobre la intervención social (seminarios de práctica).

- Encuentros mensuales de reflexión teórico-metodológica del campo de ambiente, hábitat humano y territorio.

En cuanto a las orientaciones para textualizar la práctica, se destaca la importancia de la planeación de la escritura. Para cada segmento del informe, la docente presenta los elementos mínimos que debe contener cada apartado (tabla 2).

Tabla 2. Lista de chequeo de elementos mínimos de cada apartado del informe.

\begin{tabular}{|c|c|c|}
\hline \multicolumn{3}{|c|}{$\begin{array}{l}\text { Prácticas Académicas II } \\
\text { Lista de chequeo de informes presentados - Semestre } 2019 \text { || }\end{array}$} \\
\hline Informes & Realizado & Pendiente \\
\hline Informe de análisis, revisión de la propuesta y plan de acción & & \\
\hline Diagnóstico & & \\
\hline Planeación (población objetivo, objetivos, plan de acción, cronograma) & & \\
\hline Informe referencial & & \\
\hline Marco teórico, marco conceptual, marco normativo y antecedentes & & \\
\hline Informe de ejecución & & \\
\hline Descripción del desarrollo de los objetivos & & \\
\hline $\begin{array}{l}\text { Triangulación de la información (marco referencial, resultados, análisis } \\
\text { propio) }\end{array}$ & & \\
\hline Alcances (de la intervención) & & \\
\hline Limitaciones (de la intervención) & & \\
\hline Informe de evaluación & & \\
\hline Evaluación de la intervención desde los participantes & & \\
\hline Ventajas y logros (a nivel profesional/a nivel institucional) & & \\
\hline Desventajas y dificultades (a nivel profesional/a nivel institucional) & & \\
\hline
\end{tabular}


Prácticas Académicas II

Lista de chequeo de informes presentados - Semestre 2019 II

\begin{tabular}{|l|l|l|}
\hline Informe final & & \\
\hline$\cdot$ Introducción & & \\
\hline$\cdot$ Marco referencial & & \\
\hline$\cdot$ Caracterización institucional & & \\
\hline$\cdot$ Antecedentes de prácticas anteriores & & \\
\hline$\cdot$ La práctica de trabajo social (el TS en la institución, el rol del TS en & & \\
\hline la institución, expectativas de intervención) & & \\
\hline$\cdot$ Diagnóstico & & \\
\hline$\cdot$ Planeación (población objetivo, objetivos, plan de acción, & \\
\hline cronograma) & & \\
\hline - Ejecución y análisis del proceso (incluir elementos del informe & & \\
\hline mencionado arriba) & & \\
\hline$\cdot$ Evaluación (incluir elementos del informe mencionado arriba) & & \\
\hline$\cdot$ Conclusiones & & \\
\hline$\cdot$ Recomendaciones & & \\
\hline$\cdot$ Bibliografía & & \\
\hline$\cdot$ Anexos & & \\
\hline
\end{tabular}

Fuente: elaboración propia.

La planificación textual es uno de los elementos que permite al estudiante estructurar el discurso. En general, el proceso de estructuración de un texto comprende la planificación, la escritura, la revisión y la corrección (Figueroa y Simón, 2011). Al respecto, Carlino (2002) afirma que

es necesario que [la universidad] se ocupe de enseñar a planificar y a revisar lo escrito anticipando el punto de vista del destinatario, de modo que en este proceso no solo se mejore el producto sino que se ayude a sus autores a poner en práctica el escribir como herramienta para pensar los contenidos de cada materia. (Carlino, 2002, p. 5)

Por lo anterior, el proceso de planificación, elaboración y revisión de los informes se compone de la construcción de un esquema del texto, la realización del informe, la revisión y retroalimentación docente, la revisión y retroalimentación grupal, el ajuste del informe y la retroalimentación docente con calificación cuantitativa (figura 2). 
Figura 2. Proceso de planificación, elaboración y revisión de la escritura.
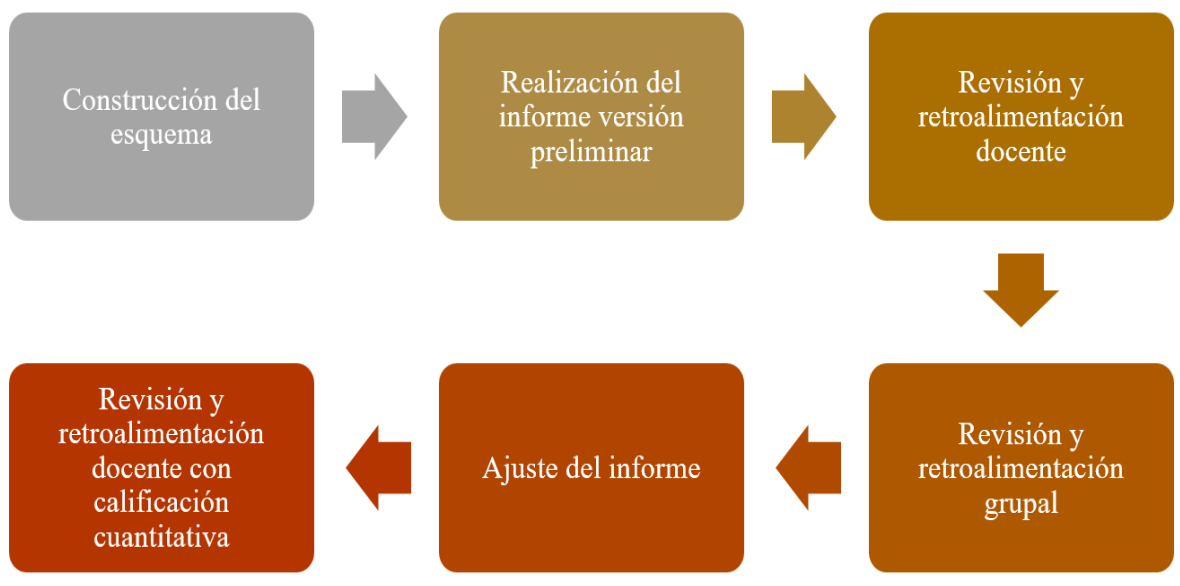

Fuente: elaboración propia.

Adicional a las estrategias anteriores, durante el trabajo de campo el docente acompaña la ejecución de actividades con los estudiantes y una vez finalizada cada jornada se reflexiona respecto a tres aspectos:

1. La realidad social, los intereses de la organización y la universidad.

2. Los fundamentos teórico-metodológicos de la acción profesional.

3. Los retos de la intervención profesional.

En síntesis, el proceso de orientación de la práctica profesional se proyecta como un escenario que busca permitir la cualificación del estudiante para el fortalecimiento tanto de las bases teóricometodológicas, como de la reflexión ético-política y técnico-operativa de la profesión (Ramos y Santos, 2016). Esto, en gran medida desde los ideales, busca hacer resistencia al proyecto de mercantilización de la educación instaurado en la academia. 


\section{La experiencia de orientación}

Orientar la práctica profesional en trabajo social es un ejercicio complejo dado que plantea diversos retos para la docencia. Esto se debe en gran medida a que las acciones pedagógicas deben ir más allá de las conferencias magistrales y las estrategias de participación tradicional, como lo son los talleres, el análisis de casos y las exposiciones, propios de la enseñanza tradicional en el aula. Por lo tanto, demanda gran capacidad reflexiva sobre las diversas fuerzas que interactúan entre los agentes y la institucionalidad. De esta manera,

todo cuanto se genera a través de la docencia y la producción de conocimiento, debe contribuir al incremento del capital social mediante el establecimiento de una relación dinámica entre la práctica docente y la reflexión que se deriva del ejercicio de enseñanza (Oppel, 2005), relación dinámica de afectación mutua tanto del profesor como del estudiante y de reconocimiento de las diversas complejidades, y de los problemas que debe enfrentar la educación. (Rojas, Patiño y Linares, 2012, p. 70)

Las primeras sesiones con los estudiantes están cargadas de expectativas respecto a su desempeño y a la institución en que se desarrollará la práctica. Para el caso descrito, se orientan tres estudiantes, quienes manifiestan haber escogido esta opción institucional por la posibilidad que brinda de realizar trabajo de campo. Durante las primeras semanas, en el acercamiento institucional se deberá reconocer las estructuras organizativas, pero a su vez se orienta sobre la necesidad de contrastar el surgimiento de la institución con el contexto social, político y económico en que se adelantan las prácticas.

Es común que al inicio las estudiantes adquieran de manera rápida el discurso institucional, lo cual se debe en gran medida a las sesiones de inducción que realiza la organización para presentar su trabajo. En ocasiones, la orientación docente gira en torno a 
cuestionar esos mismos discursos con el ánimo de desarrollar esa capacidad crítica en las estudiantes sobre el actuar organizacional, a fin de construir una mirada propia.

Con los días, las estudiantes se apropian de las dinámicas institucionales y se vinculan sin mayor problema a las actividades que se programan. Cabe resaltar que al inicio de la práctica esta vinculación se asume como un apoyo logístico, visión de la que con el tiempo tratan de desprenderse para realizar la propuesta de intervención. Asimismo, al inicio de la práctica profesional existe la incertidumbre en el estudiante sobre cómo definir lo específico, lo propio de su acción profesional, de demarcar los espacios de las demás profesiones y la propia. Esta búsqueda lleva en gran medida a perder de vista la riqueza de la interdisciplinariedad que provee el campo.

Adicional a lo anterior, durante las sesiones iniciales de orientación individual las dudas más recurrentes giran en torno a la escritura del informe de ubicación y la necesidad de revisión de los avances en este aspecto. A nivel general se encuentra gran dificultad para la construcción de ejercicios textuales que vayan más allá de la descripción de las características generales de la institución. Por esta razón, se pide a las estudiantes que a partir de los hitos institucionales, gestionen información respecto al contexto nacional y regional. Esta información la producen con base en fuentes secundarias o primarias. Existe una gran tendencia a utilizar entrevistas semiestructuradas y diálogos informales con miembros de la organización.

Los ejercicios de escritura iniciales tienen la particularidad de ceñirse a la lista de comprobación de los elementos mínimos requeridos. Por esta razón, durante la orientación se construye con las estudiantes la pregunta sobre la cual se basará su disertación y se realiza un esquema inicial del texto, compuesto por tres segmentos básicos: introducción, argumentación y conclusión. La preocupación sobre la asignación de la calificación es evidente, se busca entonces garantizar mayores revisiones para que se vaya afianzando el texto. A su vez, se recomienda la búsqueda de lec- 
tores adicionales que permitan retroalimentar la escritura y se generan espacios para la retroalimentación grupal. En general, de las observaciones grupales se destaca la necesidad de:

- Recuperar experiencias de práctica de trabajo social anteriores en la institución.

- Profundizar en el contexto regional y nacional del surgimiento de la organización.

- Mejorar la coherencia entre la introducción y las conclusiones del texto.

Luego de ello, se ajustan los informes y se entregan para calificación por parte de la docente. La calificación se realiza mediante rúbricas de evaluación, en donde adicional a la valoración de aquello que se ha escrito, se ponderan las competencias procedimentales, axiológicas y actitudinales. La entrega de la nota se realiza en un escenario de acompañamiento individual en el que se destacan las potencialidades y cuestiones por mejorar.

En este punto, dado que el estudiante ha identificado un posible objeto de intervención, se procede a construir el marco referencial. Este apartado inicia con un momento de estudio colectivo de una obra que reúna criterios de pertinencia para el campo y se prioriza la producción de conocimiento de trabajo social.

Se estudian entonces dos artículos de investigación desde la profesión: "Del paradigma mecanicista al ecológico desde Trabajo Social" de Amorocho (2009), y "Escenarios y perspectivas de Trabajo Social en ambiente" de Liévano (2013). El primero ofrece una reflexión en torno a los dilemas epistemológicos y de método, en la que se marca el acento en la importancia del paradigma ecológico emergente que no solo permite el conocimiento desde la complejidad, sino a su vez, su intervención; mientras que el segundo retoma la trayectoria de la profesión en el área ambiental, partiendo de la crisis ambiental desde la que se constituyen tres escenarios de intervención: sociedad-cultura, naturaleza y territorio. Adicional a lo anterior, se escoge la obra del profesor Arturo Escobar titulada Sentipensar con la tierra: nuevas lecturas sobre desarrollo, territo- 
rio y diferencia, dado que en ella se discute el desarrollo como un discurso que moldea la relación entre el ser humano y el territorio que se evidencia en la realidad social.

Tras escoger el material, se propone un orden. En los tres primeros encuentros se estudia el libro del profesor Arturo Escobar. En el tercer encuentro se adiciona el documento de la profesora Amanda Amorocho, y finalmente, el de la profesora Adriana Liévano, que permite la identificación de los escenarios de intervención. Para cada encuentro se llevan preguntas sobre el texto a profundizar.

Aunado a la bibliografía general, tras la entrega del informe de ubicación se identifican con cada estudiante líneas de profundización específicas y se provee material. La experiencia docente permite al estudiante navegar por bibliografía especializada. No obstante, se debe brindar herramientas para que aprenda a gestionar información de calidad, razón por la cual se organiza un taller de gestión de información secundaria, específicamente sobre bases de datos (bases de datos públicas: Clacso, Dialnet, ScieIo, Redalyc; bases de datos privadas: Scopus, Jstor; repositorios: universitarios, Intelligo), software para el análisis de citaciones académicas (software: Publish or Perish) y gestores de referencia bibliográficos (Mendeley).

A medida que se va avanzando en la lectura, se inicia la escritura de los apartados sobre el referente teórico-conceptual y el referente normativo. Para cada uno de ellos se realiza este procedimiento: identificación de elementos, planificación textual, composición escrita, revisión y retroalimentación docente, revisión y retroalimentación grupal, ajuste y revisión, y retroalimentación docente con calificación cuantitativa.

Del proceso de composición teórico-conceptual se observa en general que las estudiantes tienen elementos conceptuales con los que dialogan y establecen posturas. No obstante, entre los retos se plantea: 
- Buscar mejores estrategias para la articulación del texto.

- Realizar mayor profundización teórica para la construcción del apartado, pues en ocasiones se vinculan elementos teórico-conceptuales que, aunque son de gran riqueza interdisciplinar, no son coherentes entre sí.

- Reducir el uso de citas textuales.

En este punto cabe destacar que el marco referencial se convierte en un arma de doble filo para la intervención, dado que la teoría permite develar, pero también ocultar, y al momento el acercamiento a la realidad de intervención es limitado.

Los retos de la articulación teórica con la práctica se evidencian especialmente en el momento de diagnóstico. En este punto las estudiantes se enfrentan a identificar el tipo de diagnóstico más adecuado, acorde a su marco referencial, y los tiempos académicos, comunitarios e institucionales. Esto en particular genera tensiones entre el deseo de los procesos participativos que resultan atractivos, pero a su vez complicados por la confluencia de estos cuatro elementos.

Para la selección del tipo de diagnóstico se realiza una sesión docente grupal en donde se presentan las características de cada uno, se revisa el tiempo de ejecución y se selecciona el que, a criterio del estudiante y con orientación docente, se considera más pertinente. La tendencia general es que se realicen diagnósticos sociales con componentes participativos, pero esta participación por lo general es guiada y no protagonista, dado que los proyectos en los que se vinculan las estudiantes surgen desde un contexto externo al que se interviene y no especialmente de los procesos de organización de base.

Una vez se escoge el diagnóstico, se realiza su conceptualización y se crean los instrumentos y técnicas de recolección de información. Por lo general, en este punto se acude a la investigación para dar cuenta de la situación de las comunidades. Para el diseño 
de técnicas e instrumentos, se realiza un taller grupal. Para el caso, resultó necesaria la orientación docente frente a la construcción de:

- Entrevistas o diálogos semiestructurados y consentimientos informados.

- Encuestas (identificación devariablesyoperacionalización).

- Diseño de técnicas interactivas acordes a los tiempos y el acceso a campo.

En este diseño es fundamental la identificación de actores, así como el énfasis en las convocatorias para los encuentros colectivos. En dos casos las estudiantes decidieron realizar primero el encuentro colectivo para desde allí identificar actores clave, y en el otro la estudiante realizó el procedimiento desde lo individual a lo colectivo.

Una vez se realiza el diseño, las estudiantes acceden al campo y con la información recolectada se encuentran listas para su análisis. En este momento, la docente realiza dos encuentros grupales y uno individual para iniciar la organización y crítica de la información. Durante el primer encuentro se orienta el análisis cualitativo mediante un software especializado (Atlas.ti, versión 7.1); en el segundo encuentro se orienta el análisis cuantitativo (IBMSPSS, versión 21), y en el encuentro individual se realiza el esquema de planificación textual. De igual forma, estos documentos son revisados y retroalimentados en escenarios individuales (docenteestudiante) y grupales (docente-estudiantes). Entre las principales dificultades de este momento de la práctica se encuentran:

- Las tensiones por el desfase entre los tiempos comunitarios, institucionales y académicos.

- La frustración del estudiante ante lo planeado y lo ejecutado. 
De lo anterior cabe destacar que las tensiones se deben principalmente a las reflexiones propias de los estudiantes, dado que todo el tiempo se cuestionan por su actuar y su contribución al proyecto ético-político de la profesión, el cual debe trascender de la ejecución de actividades para el logro de las metas. En continuas oportunidades cuestionan a la universidad y al docente por ceñirse a los tiempos y entregables, así como a la institución de la práctica por no mantener una presencia prolongada en el campo, que se limita a la disponibilidad de recursos.

A la par del diagnóstico se realiza la planeación. A pesar del interés de los estudiantes por respetar los tiempos comunitarios, nuevamente se incurre en la realización de ejercicios de planificación tradicionales realizados solo por el profesional social. De esta manera, la participación comunitaria se restringe a tener el conocimiento sobre lo que este experto, externo, considera que es necesario para solucionar una situación problema. Al finalizar la Práctica I, las expectativas de los estudiantes han cambiado, la docencia se ve ante una encrucijada por resistir al modelo de competencias instaurado por la política nacional de educación y la distorsión de las instituciones sobre la práctica del trabajador social, que en ocasiones asigna actividades desvinculadas de la profesión y ve al practicante como mano de obra barata, en una dinámica en la que no se asume la corresponsabilidad de la formación.

Infortunadamente, el desarrollo de la segunda práctica no es diferente al anterior. A pesar de la evaluación institucional y los compromisos que se asumen, las tensiones entre las fuerzas de los agentes (estudiantes y docentes) y la institucionalidad (unidad académica e institución de práctica) se mantienen. Esto genera que la intervención de los estudiantes se reduzca a los espacios de encuentro colectivos, en donde los talleres parecen ser la única herramienta para "cumplir" con los requisitos de la práctica. Al respecto, Bermúdez (2011) afirma que:

Las decisiones en el campo de fuerzas de la intervención representan de alguna manera los marcos burocráticos 
en los que ésta se mueve: maneras de operar, mecanismos de relación y control (como contratos), términos de referencia, etc., que serían algunas de las formas a través de la cuales se expresan las decisiones administrativas. Lo interesante de este aspecto es que no interviene un único vector en la toma de decisiones. (p. 88)

La evaluación de la práctica deber permitir al estudiante reflexionar sobre estas cuestiones y proponer estrategias para la ruptura de acciones que perpetúan el habitus, caracterizado hasta ahora por ser perpetuador de las relaciones hegemónicas de dominación. No obstante, en este punto los estudiantes se encuentran agotados y parecen haberse adaptado a la producción de resultados, productos y evidencias. En este punto el docente realiza, mediante encuentros individuales y colectivos, reflexiones sobre el proyecto ético-político de la profesión, el alcance de la práctica, y los retos individuales e institucionales.

\section{Interpretación y potenciación de la experiencia}

La intervención social es un campo de análisis y acción que, aunque no es exclusivo del trabajo social, es un rasgo distintivo de la profesión. De hecho, la intervención social es objeto de conocimiento y de acción (Bermúdez, 2011). Por tal razón es fundamental el rol docente en la orientación de las prácticas preprofesionales de los trabajadores sociales, en la medida en que permite no solo aportar a la construcción de la identidad profesional de los estudiantes, sino que a su vez busca que se asuma la acción como un ejercicio reflexivo y crítico de los conocimientos teóricometodológicos del trabajo social.

Los aspectos a destacar de la orientación docente durante la práctica académica se basan en la selección de material bibliográfico para el estudio individual y colectivo. Asimismo, la identificación de las macro- y microestructuras del género textual de 
los informes de práctica puede verse como una ayuda para el ejercicio de textualización por parte de los estudiantes.

Respecto a la asesoría de la profesora en cuanto a lecturas, ha sabido guiarlas de manera que recomienda textos o conceptos que abordan y relacionan la complejidad de este tema y cuando nos encontramos en espacios de debate y análisis de la práctica lo sabe relacionar también con el quehacer profesional y la ética del trabajo social, esto ayudándose también de herramientas como el checklist que creó para el proceso y los documentos que ha dejado en el repositorio virtual. (DC8_05032019_Estudiante1)

Estar en práctica me ha conducido a fortalecer competencias como la lectura y la escritura de textos académicos, pues si bien la lectura me ha resultado muy enriquecedora, en el momento de la redacción de los informes he tenido algunas dificultades de organización, de redacción, y de identificación de fuentes. Sin embargo, la forma en la que la profesora planteó la estructura y escritura de los informes ha contribuido para que la expresión de las ideas fluya de una manera más armónica y la lectura resulte más amena. (DC22_11062019_Estudiante2)

No obstante, el hecho de realizar una introducción teórica y determinar la necesidad de construir un marco referencial sin tener un acercamiento por parte del estudiante a la realidad social es una práctica positivista que sesga la visión del contexto. Asimismo, el brindar estructuras textuales de forma tan esquemática implica que el estudiante, con el transcurrir de su práctica, asuma la escritura no como un proceso de reflexión, sino como un asunto técnico en el que interesa completar los apartados que se han solicitado, muchas veces sin que estos tengan algún sentido. De esta manera, podría decirse que la escritura, más allá de ser liberadora, adoctrina para el mercado laboral. 
Por lo tanto, es importante destacar que, aunque el proyecto educativo se basa en la formación por competencias, la resistencia desde la docencia al proyecto mercantilizador de la educación debe basarse en la posibilidad de potenciar en los estudiantes competencias críticas. Al respecto, lamamoto (2003) afirma que el rumbo ético-político contemporáneo del trabajo social

requiere un profesional informado, culto, crítico y competente. Exige romper tanto con el teoricismo estéril como con el pragmatismo que deja prisionero al profesional en el hacer por hacer, pensando en metas e intereses inmediatos. Demanda competencia, pero no la competencia autorizada y permitida, la competencia de la organización que diluye el poder como si no fuera ejercido por nadie, como si derivara de las "normas" de la institución, de la burocracia. El requisito es otro, se trata de una competencia crítica capaz de descifrar la génesis de los procesos sociales, sus desigualdades y las estrategias de acción para enfrentarlas. Supone competencia teórica y fidelidad al movimiento de la realidad, competencia técnica y ético-política que subordine el "cómo hacer" al "qué hacer" y este al "deber ser", sin perder de vista sus raíces en el proceso social. (lamamoto, 2003, p. 100)

Esto puede ser posible si, entre otras acciones, se reestructura la práctica y se exploran nuevos géneros textuales. Quizá resulte más satisfactorio el acercamiento a la realidad y la ubicación de un problema práctico que luego debe ser teorizado, y sobre el cual se deben identificar las causas de la estructura social de dominación que permiten que este perdure para transformarlo; en lugar de partir de lo que plantea la teoría acerca lo que debe ser un territorio o el desarrollo rural para luego pasar a comprobar si la realidad cumple o no con lo que se tiene establecido. No obstante, ello no implica asumir que la práctica y la teoría se oponen; por el contrario, deben ser dos cuestiones paralelas a la intervención profesional.

El Servicio Social debe reconocer como práctica profesional no solo aquello cuyo proceso completo es realizado 
por el mismo individuo. El proceso debe ser desarrollado por la profesión como un todo y no necesariamente por cada profesional particular. No se debe despreciar la actividad del "profesional de campo" por considerarla limitada teóricamente, ni se debe deslegitimar las elaboraciones teóricas de un "profesional académico" acusándolo de no estar sostenidas en la experiencia práctica. Este enfrentamiento mutuo trajo experiencias nocivas al Servicio Social, reproduciendo la lógica positivista de su génesis, donde la producción de conocimiento es cuestión de las "ciencias" y al asistente social corresponde su aplicación en la práctica. (Montaño, 2000, p. 143)

En este escenario, la investigación cumple un rol protagónico. Indudablemente, la práctica académica se relaciona e integra a la investigación (Vasconcelos, 2009). En ella, el estudiante se ve en la necesidad de movilizarse para realizar la identificación de problemas propios de las realidades sociales que interviene. No obstante, dadas las tensiones entre las fuerzas presentes entre los agentes y la institucionalidad, la investigación se convierte en una herramienta positivizada, que va de lo deductivo a lo inductivo, en la cual se debe dar cuenta del "cómo" de manera detallada para obtener resultados confiables y válidos.

A pesar de lo anterior, durante la práctica se brindaron herramientas que buscan que los estudiantes sean autónomos en el aprendizaje. Para ello fue necesaria la orientación en gestión documental, gestión de información primaria, análisis e interpretación de datos. Al respecto, los estudiantes se refieren así:

Un punto que yo resalto mucho es el repaso que se realizó de bases de datos, tanto de la universidad, como externas y de programas de gestión documental como Mendeley y Publish or Perish, las cuales me han sido de bastante ayuda para la búsqueda y referenciación bibliográfica, demasiado interesante este hecho. (DC8_05032019_Estudiante1) 
Me gustó mucho cuando nos reunimos entre todas a analizar los datos, esto me permitió reforzar lo que había aprendido en el aula y que la interpretación de mi caso fuera retroalimentada por mis compañeras; ellas vieron cosas que yo no pude ver y eso me permitió realizar una propuesta de intervención más adecuada. (DC27_04052019_Estudiante3)

Como se observa en estos testimonios, las estudiantes valoraron como significativos los escenarios de construcción colectiva. Allí los estudiantes tejen solidaridades y afinan sus apuestas de intervención; asimismo se da el espacio para reflexionar sobre la acción.

El acompañamiento y las orientaciones que he recibido de la profesora además de pertinentes han sido fundamentales para que este reto sea más llevadero y enriquecedor en mi vida académica y personal, pues aunque hemos trabajado de modo individual, siento que la mayor riqueza ha estado en la invitación a construir de manera colectiva, a trabajar en equipo, a conocer el proceso de la compañera y hacer parte de él, pues si bien en toda la carrera he trabajado en grupo, en pocas ocasiones me he sentido parte de este, contrario a este momento. (DC19_06112019_Estudiante2)

El relato anterior lleva a cuestionarse acerca de la especificidad y la interdisciplinariedad. La búsqueda por lo específico lleva a cerrar posibilidades de discusión no solo con profesionales sino a su vez con las comunidades locales, pues el trabajador social se erige como experto que durante la práctica configura su objeto de intervención, en ocasiones aislado de una retroalimentación desde su área de formación en consonancia con otras disciplinas. Durante la orientación se destacó la importancia del trabajo interdisciplinario, en particular para el campo de ambiente, territorio y hábitat humano.

Ya enfocándome en mi proceso de práctica, me llama muchísimo la atención la interdisciplinariedad que se ma- 
neja en todos los ámbitos de este tema, lo que hace que siempre sea complejo y enriquecedor cualquier tipo de acercamiento. Por ejemplo, cuando empecé a leer acerca de lo relacionado al campo, me encontré con que hay diversas perspectivas que van más allá de una relación estricta con la naturaleza y el ambiente, pues si bien en esto se basa, hay relación política, económica e histórica. Siempre hay un macroanálisis de una situación específica. Además, la profesora en las orientaciones me llevó a encontrar que han sido bastantes los autores que se han encargado de justificar estos temas desde la teoría, especialmente en América Latina, dándole una aplicación local a los conocimientos rescatados desde las mismas poblaciones y analizándolo a la luz de algunos otros teóricos. (DC32_09172019_Estudiante3)

La mayor tensión que se da en la práctica responde a la apuesta del modelo educativo por competencias que acoge la universidad, y por ende, la Escuela de Trabajo Social, para dar cumplimiento a los estándares de calidad en la formación profesional. Los estudiantes expresan este problema como una dificultad para la articulación entre las demandas académicas, institucionales y las realidades sociales.

[...] una de las cosas que considero que debe tenerse en cuenta en la práctica es el manejo del tiempo a nivel interno en comparación al externo, es decir, lo establecido por la escuela, lo que pide la organización y lo que pasa en la comunidad. Los tiempos de la universidad nos presionan y aunque la profesora es flexible, en ocasiones por cumplir se hace un trabajo poco consciente y con poca disposición. (MC7_28082019_Estudiante2)

Esta situación conlleva que el profesional se adapte a las necesidades de mercado que demanda la demostración del cumplimiento de actividades, metas e indicadores. Con el tiempo, las apuestas de intervención reducen la participación comunitaria a la asistencia en espacios direccionados por el practicante. Esto se acentúa cuan- 
do las instituciones donde se desarrolla la práctica, especialmente las organizaciones no gubernamentales, carecen de recursos que permitan la permanencia prolongada en el campo y conciben al practicante como mano de obra barata y calificada que les permite cumplir metas a corto plazo en las comunidades en que intervienen.

Llama la atención que, aunque durante el proceso de formación es el estudiante quien cuestiona la intervención, a la institución y a la universidad a partir del proyecto ético-político profesional ideado, con el transcurrir de la práctica se adapta a la lógica del proyecto educativo mercantilizado. Hoy en día el mayor reto para transformar el habitus es que la orientación docente promueva al interior de la unidad académica la discusión en torno a las competencias y el sistema de evaluación creado para las prácticas académicas, así como el análisis constante del contexto sociopolítico contemporáneo. Asimismo, se destaca la importancia de sistematizar el rol del docente en la formación profesional de trabajadores sociales a fin de realizar ejercicios críticos reflexivos que tengan un efecto transformador de los problemas, atendiendo la causa de la estructura social. De igual manera, es fundamental que las unidades académicas de trabajo social realicen apuestas de intervención social no solo desde las instituciones, sino que se creen laboratorios sociales que permitan desarrollar un proceso de desarrollo comunitario en el tiempo.

\section{Referencias bibliográficas}

Acevedo, M. (2008). La metáfora de los escenarios de educación popular como dispositivo de interpretación de experiencias. Revista Internacional Magisterio, 33, 24-31. Recuperado de http://bibliotecadigital.magisterio. co/revista/no-33-sistematizaci-n-de-experiencias

Alexopoulou, A. (2011). El enfoque basado en los géneros textuales y la evaluación de la competencia discursiva. En J. de Santiago, H. Bongaerts, 
J. Sánchez y M. Seseña (eds.), Del texto a la lengua: la aplicación de los textos a la enseñanza-aprendizaje del español L2-LE (volumen I, pp. 98110). Salamanca: Universidad de Salamanca.

Bermúdez, C. (2011). Intervención social desde el Trabajo Social: un campo de fuerzas en pugna. Prospectiva, 16, 83-101. https://doi.org/ https://doi.org/10.25100/prts.v0i16.1164

Bourdieu, P. y Wacquant, L. (2005). Una invitación a la sociología reflexiva. Buenos Aires: Siglo XXI. Recuperado de https://sociologiaycultura.files. wordpress.com/2014/02/bourdieu-y-wacquant.pdf

Carlino, P. (2002). Enseñar a planificar y a revisar los textos académicos: haciendo lugar en el currículum a la función epistémica de la escritura. Artículo presentado en las IX Jornadas de Investigación, Facultad de Psicología, Universidad de Buenos Aires. Recuperado de https://www. aacademica.org/paula.carlino/27.pdf

Corredor, M., Arbeláez, R. y Pérez, M. (2009). Estrategias de enseñanza y aprendizaje. Bucaramanga: Universidad Industrial de Santander.

Díaz, Á. (2013). Secuencias de aprendizaje. ¿Un problema del enfoque de competencias o un reencuentro con perspectivas didácticas? Profesorado: Revista de Currículum y Formación del Profesorado, 17(3), 11-33. Recuperado de https://www.redalyc.org/pdf/567/56729527002. pdf

Escuela de Trabajo Social (Universidad Industrial de Santander). (2011). Reforma curricular del programa de Trabajo Social. Bucaramanga. (Documento Word)

Figueroa, R. y Simón, J. (2011). Planificar, escribir y revisar, una metodología para la composición escrita: una experiencia con estudiantes del Instituto Pedagógico de Caracas (IPC). Revista de Investigación, 35(73), 119-148.

lamamoto, M. (2003). El servicio social en la contemporaneidad (C. Montaño, ed.). São Paulo: Cortez. 
Jara, O. (2018). La sistematización de experiencias: práctica y teoría para otros mundos políticos. Bogotá: Centro Internacional de Educación y Desarrollo Humano (CINDE). Recuperado de https://repository.cinde. org.co/bitstream/handle/20.500.11907/2121/Libro sistematización Cinde-Web.pdf?sequence $=1 \&$ isAllowed $=y$

Montaño, C. (2000). La naturaleza del servicio social: un ensayo sobre su génesis, su especificidad y su reproducción (2. ed.). São Paulo: Cortez.

Montaño, C. (2007). Trabajo social e intervención: la politización de la acción profesional. Conferencia presentada en el VII Coloquio Internacional de Estudiantes de Trabajo Social: Desarrollo, Política Social e Intervención Profesional. Universidad del Altiplano, Puno, Perú. Recuperado de http://www.ts.ucr.ac.cr/binarios/pela/pl-000360.pdf

Parola, R. (2009). Producción de conocimiento en el trabajo social: una discusión acerca de un saber crítico sobre la realidad social. Buenos Aires: Espacio.

Ramos, A. y Santos, F. E. Coutinho dos. (2016). Articulação entre supervisão de campo e acadêmica em Serviço Social. Temporalis, 16(31), 281-303. Recuperado de http://periodicos.ufes.br/temporalis/article/ view/12301

Rodríguez, C., Lorenzo, O. y Herrera, L. (2005). Teoría y práctica del análisis de datos cualitativos: proceso general y criterios de calidad. Revista Internacional de Ciencias Sociales y Humanidades, SOCIOTAM, XV(2), 133-154.

Rojas, M., Patiño, L. y Linares, J. (2012). La docencia expuesta: las prácticas pedagógicas en la universidad. Bucaramanga: Universidad Industrial de Santander

Vasconcelos, I. (2009). Dilemas e desafios do estagio curricular em Serviço Social: expressão dos (des)encontros entre a formação profissional e o mercado de trabalho. Temporalis, 9(17), 61-82. 